\title{
14-kDa Phosphohistidine phosphatase plays an important role in hepatocellular carcinoma cell proliferation
}

\author{
SU-XIA HAN, LI-JUAN WANG, JING ZHAO, YING ZHANG, MENG LI, XIA ZHOU, JING WANG and QING ZHU \\ Department of Oncology, The First Affiliated Hospital, College of Medicine, \\ Xi'an Jiaotong University, Xi'an, Shaanxi 710061, P.R. China
}

Received March 22, 2012; Accepted July 4, 2012

DOI: $10.3892 / \mathrm{ol} .2012 .802$

\begin{abstract}
Da Phosphohistidine phosphatase (PHP14), the first histidine phosphatase protein identified in vertebrates, was recently revealed to play an essential role in lung cancer. The function of this gene in other tumors is unclear; however, in this study, we demonstrate that PHP14 is highly expressed in hepatocellular carcinoma (HCC) tissues and cell lines compared with adjacent non-cancerous human liver tissues and cells $(\mathrm{P}<0.05)$. We used lentivirus-mediated delivery of small interfering RNA (siRNA) to knockdown the expression of PHP14 in an HCC cell line and investigate the effects of PHP14 on cell growth in vitro. Cell proliferation was inhibited and cell apoptosis was significantly increased. PHP14-siRNA affected the cell cycle and promoted $\mathrm{G} 1 \rightarrow \mathrm{S}$ phase transition in HCC cells. These results demonstrate that the knockdown of PHP14 expression by lentivirus-delivered siRNA may be a useful therapeutic approach for the treatment of HCC.
\end{abstract}

\section{Introduction}

Hepatocellular carcinoma (HCC) is the 5th most common type of malignancy worldwide $(1,2)$. Each year, there are 500,000 to 1 million new cases worldwide, particularly in Asia and Africa (3). Although the prognosis of HCC has improved due to new advances in treatment, the overall survival of patients with HCC remains unsatisfactory $(4,5)$. Following surgery, the 5-year survival rate is limited to $25-39 \%$ (6), and is much lower

Correspondence to: Dr Qing Zhu, Department of Oncology, The First Affiliated Hospital, College of Medicine, Xi'an Jiaotong University, No. 277 Yanta West Road, Xi'an, Shaanxi 710061, P.R. China

E-mail: newzhuqing1972@yahoo.com

Abbreviations: HCC, hepatocellular carcinoma; PHP14, 14-kDa phosphohistidine phosphatase; mRNA, messenger RNA; MOI, multiplicity of infection; PCR, polymerase chain reaction; PBS, phosphate-buffered saline; RNAi, RNA interference; siRNA, small interfering RNA

Key words: hepatocellular carcinoma, PHP14, proliferation, cell cycle, apoptosis elsewhere $(7,8)$. The aggressive nature of $\mathrm{HCC}$ is associated with mutations of various oncogenes and tumor-suppressor genes; therefore, novel therapeutic targets for HCC are required (9). Advances in HCC treatment are likely to require a more detailed understanding of its biology and behavior; thus, the mechanisms underlying the rapid metastatic capacity of HCC require further study $(10,11)$.

The 14-kDa phosphohistidine phosphatase (PHP14), also known as PHPT1, was the first histidine phosphatase protein identified in vertebrates and is similar to the janus proteins of Drosophila $(12,13)$. PHPT1 demonstrates specific phosphatase activity against peptides and proteins containing phosphohistidine (14). Studies investigating the physiological function of PHP14 have revealed that PHP14 is able to dephosphorylate ATP-citrate lyase (15) and the $\beta$-subunit of $\mathrm{G}$ proteins in vitro (16). Among the total number of phosphorylations in eukaryotes, only $7 \%$ are histidine phosphorylations and they may be involved in the signal transduction of cancer $(17,18)$. Klumpp et al revealed that PHP14 may play a role in neuronal signal transduction (17). It has also been suggested that PHP14 plays a role in human lung cancer cell migration and invasion (19); however, the role of PHP14 in HCC remains unknown.

In the present study, we confirmed that PHP14 is highly expressed in HCC tissues and cell lines. Additionally, we used the newly developed lentivirus-mediated delivery of small interfering RNA (siRNA) technique (20) to observe the effects of PHP14 knockdown on human HCC cell growth and apoptosis in vitro.

\section{Materials and methods}

Cell lines and culture. The HCC cell lines HepG2 and SMMC7721 were preserved at the Department of Oncology at The First Affiliated Hospital, Xi'an Jiaotong University, China. Human liver HL-7702 cells were purchased from the American Type Culture Collection (Manassas, VA, USA). Cells were cultured in RPMI-1640 medium supplemented with $10 \%$ fetal bovine serum (FBS), $100 \mathrm{U} / \mathrm{ml}$ penicillin and $100 \mu \mathrm{g} / \mathrm{ml}$ streptomycin, in a humidified incubator with $5 \% \mathrm{CO}_{2}$ at $37^{\circ} \mathrm{C}$. All cells were passaged twice each week and routinely examined for mycoplasma contamination. Cells in the logarithmic growth phase were used for further experiments. 
Tissue collection and immunohistochemistry. Primary site HCC tissues, adjacent benign liver tissues and normal hepatic tissues were obtained from 38, 37 and 31 patients, respectively. All cases of HCC were clinically and pathologically confirmed and all patients signed informed consent forms prior to surgery, which was conducted at the Department of Pathology, The First Affiliated Hospital of Xi'an Jiaotong University, China. The protocols used in this study were approved by the Hospital's Protection of Human Subjects Committee.

Sections $(5 \mu \mathrm{m})$ of formalin-fixed paraffin-embedded specimens were collected. Slides were dewaxed, rehydrated, incubated in $10 \%$ normal goat serum and $0.3 \%$ Triton X-100 in PBS for $1 \mathrm{~h}$, then incubated with monoclonal rabbit anti-PHP14 polyclonal antibody (1:150; Sigma, Swampscott, MA, USA). The slides were washed 3 times in PBS for $5 \mathrm{~min}$. The tissues were then incubated in biotin-labeled rabbit anti-mouse serum (1:200) for $30 \mathrm{~min}$, rinsed with PBS and incubated with avidin-biotin peroxidase complex for $1 \mathrm{~h}$. The signal was detected using 3,3'-diaminobenzidine as the chromogen. Negative control slides omitting the primary antibody were included in all assays and all sections were examined independently by 2 investigators.

Expression of PHP14 was evaluated according to the ratio of positive cells per specimen and staining intensity, as previously described (21). The ratio of positive cells per specimen was evaluated quantitatively and scored as follows: 0 , staining of $<1 \%$; 1 , staining of $2-25 \% ; 2$, staining of $26-50 \% ; 3$, staining of $51-75 \%$; 4 , staining of $>75 \%$. Intensity was graded as follows: 0 , no signal; 1 , weak signal; 2 , moderate signal; 3 , strong signal. A total score of 0-12 was finally calculated and graded as: negative (-), score of $0-1$; weak $(+)$, score of $2-4$; moderate $(++)$, score of 5-8; strong $(+++)$, score of 9-12.

Recombinant lentivirus generation. The complementary DNA sequence of PHP14 was designed from the full-length ZEB1 sequence by Shanghai Gene Chem Company (Shanghai, China). The potential target sequences for RNA interference (RNAi) were scanned using the siRNA Target Finder and Design Tool available on the Ambion website. The PHP14-siRNA-targeting sequence was CGCCATTTCAACTGAGAAA (19). After testing for knockdown efficiencies, the stem-loop oligonucleotides were synthesized and cloned into the lentivirus-based PsicoR vector (Addgene, Boston, MA, USA). A non-targeting (scrambled) stem-loop DNA PsicoR vector was generated as a negative control. HCC cells were infected with a ZEB1-siRNA lentivirus or a negative control virus at 7 days and examined at 10 days.

Lentivirus infection. The HCC cell lines, HepG2 and SMMC7721, were incubated with lentivirus in a small volume of serum-free DMEM at $37^{\circ} \mathrm{C}$ for $4 \mathrm{~h}$. Following this, $10 \%$ DMEM was added to the cells and they were placed in an incubator for an additional indicated time for the following experiments. Subsequently, the following experiments were conducted using viruses at a multiplicity of infection (MOI) of 20, unless indicated otherwise. HepG2 and SMMC7721 cells transfected with the PHP14-siRNA or scramble-siRNA were designated lenti-siRNA/PHP14 and src-siRNA, respectively.
Cell viability and proliferation. Cell viability was examined using routine 3-(4,5-dimethylthiazol-2-yl)-2,5-diphenyltetrazolium bromide (MTT) assay 3 days following virus infection at the indicated MOI. A cell proliferation assay was conducted by counting the number of cells. Cells were plated in triplicate at a density of $5.0 \times 10^{4}$ cells $/ \mathrm{ml}$ in 24-well plates and infected with the virus at the indicated MOIs. They were harvested daily and counted using a hemocytometer.

Colony formation assay. A soft-agar colony formation assay was conducted to assess the anchorage-independent growth ability of cells as a characteristic of in vitro tumorigenicity using a Cellomics Arrayscan (Genechem, Shanghai, China). Briefly, HepG2 and SMMC-7721 cells were infected with the virus for $24 \mathrm{~h}$. The cells were then detached and plated in 6 -well plates $\left(1.0 \times 10^{4}\right.$ cells/well) containing $0.3 \%$ agarose and a $0.5 \%$ agarose underlay. The number of foci $(>100 \mu \mathrm{m})$ was counted after 17 days. Each experiment was conducted in triplicate.

Flow cytometry analysis. Flow cytometry analysis was used to determine the distribution of the cells in the cell cycle and the cells undergoing apoptosis. Briefly, HepG2 and SMMC7721 cells were seeded and infected with the virus for $96 \mathrm{~h}$ in complete medium and placed in a serum-free medium for $48 \mathrm{~h}$. Once the adherent cells were collected by trypsinization, the cells were suspended in $\sim 0.5 \mathrm{ml}$ of $70 \%$ alcohol and maintained at $4^{\circ} \mathrm{C}$ for $30 \mathrm{~min}$. The suspension was filtered through a $50-\mu \mathrm{m}$ nylon mesh and the DNA content of the stained nuclei was analyzed using a flow cytometer (EPICS XL; Beckman Coulter, Inc., Brea, CA, USA). Cell cycle analysis was conducted using MultiCycle DNA cell cycle analysis software. Apoptotic cells were identified as a hypodiploid DNA peak, which represented the cells in the sub- $\mathrm{G}_{1}$ phase. Results from at least 20,000 cells were collected and analyzed using CellQuest software (BD Biosciences, Franklin Lakes, NJ, USA).

Reverse-transcription polymerase chain reaction (RT-PCR) analysis. Total RNA was extracted using TRIzol reagent (Invitrogen, Carlsbad, CA, USA) according to the manufacturer's instructions. A total of $1 \mu \mathrm{g}$ of RNA was subjected to RT. The PCR primers used for the PHP14 internal control were: sense, 5'-GCCACAGAGCCACCCCCA-3'; antisense, 5'-ATCCAGACAAATCCTTCCAGCA-3'. The PCR primers used for glyceraldehyde 3-phosphate dehydrogenase (GAPDH) were: forward, 5'-TGGTATCGTGGAAGGACTCA-3'; reverse, 5'-CCAGTAGAGGCAGGGATGAT-3' (19). PCR products were separated on a $1 \%$ agarose gel, then visualized and photographed under ultraviolet light.

Western blot analysis. Following protein quantization using a Coomassie Brilliant Blue assay, $50 \mu \mathrm{g}$ of protein was boiled in loading buffer, resolved on $10 \%$ SDS polyacrylamide gels, electrotransferred to nitrocellulose membranes and incubated overnight with mouse monoclonal antibodies against PHP14, Bcl-2, Bax (1:500; Santa Cruz Biotechnology, Inc., Santa Cruz, CA, USA) and $\beta$-actin (1:5000; Sigma, St. Louis, MO, USA). The secondary antibody (1:1000; peroxidase-conjugated anti-mouse $\operatorname{IgG}$ ) was applied, and the relative content of the target proteins was detected by chemiluminescence. 
Table I. Immunohistochemical analysis of PHP14 in HCC tissues, adjacent benign liver tissues and normal liver tissues.

\begin{tabular}{lcccccc}
\hline & \multicolumn{2}{c}{ Liver tissue, $\mathrm{n}(\%)(\mathrm{n}=68)$} & & \multicolumn{2}{c}{ HCC differentiation, $\mathrm{n}(\%)(\mathrm{n}=38)$} \\
\cline { 2 - 3 } Immunostaining & Normal $(\mathrm{n}=31)$ & Adjacent benign $(\mathrm{n}=37)$ & & Well $(\mathrm{n}=12)$ & Moderate $(\mathrm{n}=14)$ & Poor $(\mathrm{n}=12)$ \\
\hline- & $16(51.61)$ & $17(45.95)$ & & $2(16.78)$ & $2(14.29)$ & $1(8.33)$ \\
+ & $10(32.26)$ & $14(37.84)$ & & $3(25)$ & $2(14.29)$ & $2(16.78)$ \\
++ & $3(9.68)$ & $5(13.51)$ & & $3(25)$ & $3(21.43)$ & $3(25)$ \\
+++ & $2(6.45)$ & $1(2.70)$ & & $4(33.33)$ & $7(50)$ & $6(50)$ \\
\hline
\end{tabular}

Expression of PHP14 was evaluated according to the ratio of positive cells per specimen and staining intensity. PHP14 staining was graded as negative (-), score of 0-1; weak (+), score of 2-4; moderate, (++) score of 5-8; strong (+++), score of 9-12. PHP14, 14-kDa phosphohistidine phosphatase; HCC, hepatocellular carcinoma.

Statistical analysis. Statistical analysis was conducted using the Kruskal-Wallis rank test and the Mann-Whitney U test to calculate P-values and to compare the differences between the immunohistochemistry groups. Assays for characterizing cell phenotypes were analyzed using the Student's t-test. The statistical SPSS software package (SPSS, Chicago, IL, USA) was used to analyze the data. $\mathrm{P}<0.05$ was considered to indicate a statistically significant difference.

\section{Results}

PHP14 expression is elevated in the majority of HCC patient samples and cell lines. We examined the expression of PHP14 in 58 tissue samples collected from HCC patients. Immunohistochemical images revealed that the expression of PHP14 in HCC tissues was significantly higher compared with that in the adjacent non-cancerous HCC and normal liver tissues (Fig. 1A). A summary of PHP14 expression using immunohistochemical analysis in HCC tissues, adjacent benign liver tissues and normal liver tissues is presented in Table I. The average staining score of PHP14 in the HCC tissues was significantly higher compared with that of the adjacent non-cancerous HCC tissues and normal liver tissues (Fig. 1B) ( $7.53 \pm 0.78$ vs. $3.67 \pm 0.36$ vs. $3.21 \pm 0.42$, respectively; $\mathrm{P}<0.01)$. Expression of PHP14 mRNA in the 3 tissue groups confirmed the same result (Fig. 1C). PHP14 expression was clearly elevated in HCC tumor tissues compared with adjacent non-cancerous liver tissues. PHP14 mRNA expression was markedly increased in the 2 cancer cell lines, HepG2 and SMMC7721, compared with that of the human liver cell line, HL-7702 (Fig. 1D). Similarly, differential expression of the PHP14 protein was confirmed by western blot analysis (Fig. 1E). These results indicate that PHP14 expression is closely correlated with HCC.

Lentivirus-delivered siRNA knockdown of PHP14 expression and inhibition of HCC cell proliferation. To further explore the correlation between PHP14 and HCC, we constructed lentivirus-delivered vectors, a PHP14-specific siRNA vector (lenti-siRNA/PHP14) and a scramble siRNA vector (src-siRNA). The vectors were then transfected into HepG2 and SMMC7721 cells. A suppression rate of up to $80 \%$ was observed at $96 \mathrm{~h}$ in the cells infected with lenti-siRNA/PHP14. The inhibitory effect of the lenti-siRNA/PHP14 was revealed to be specific as the control and src-siRNA group had little effect on PHP14 expression levels (Fig. 2A and B).

To examine the reduction level of target mRNA induced by the lentivirus-delivered siRNAs, RT-PCR was conducted using the prepared mRNA from the infected HepG2 and SMMC-7721 cells. As shown in Fig. 2C and D, lenti-siRNA/PHP14-transfection caused a marked reduction in the level of PHP14 mRNA, while the src-siRNA-transfected and control cells did not. Altogether, these data indicated that the PHP14-siRNA suppressed the PHP14 overexpression in HCC cells effectively.

These results confirmed that lenti-siRNA/PHP14 efficiently repressed PHP14 expression. We then tested the effect of lenti-siRNA/PHP14 on the cell viability of HepG2 and SMMC7721 cells in culture. As shown in Fig. 3A, lenti-siRNA/PHP14 significantly inhibited cell growth of HepG2 and SMMC7721 cells compared with scr-siRNA-transfected and control cells. The inhibitory effect on HepG2 and SMMC7721 cell growth was also was confirmed by colony formation assays, which demonstrated that the colony-forming ability of HCC cells was significantly inhibited by transfection with lenti-siRNA/PHP14 (Fig. 3B). These results suggest that knockdown of PHP14 by lenti-siRNA/PHP14 inhibited the expression of PHP14 in HepG2 and SMMC7721 cells compared with the scr-siRNA-transfected and control group.

Lenti-siRNA/PHP14 inhibits cell cycle S phase entry of HCC cells. We examined the effect of lenti-siRNA/PHP14 on cell viability of HepG2 and SMMC7721 cells in cell culture. The human liver cell strain HL-7702, infected with the virus at a MOI of 20, was taken as a negative control. As shown in Fig. 3C, following virus infection, lenti-siRNA/PHP14 significantly inhibited cell growth of both HCC cell lines compared with the control group. The difference was more pronounced after day 3 , a time in which essentially all the cells established cell-cell contacts. The inhibitory effects on HCC cell growth were also confirmed by colony formation assays, which demonstrated that the colony-forming ability of HCC cells was significantly inhibited by transfection with lenti-siRNA/PHP14.

Flow cytometry was conducted $96 \mathrm{~h}$ after infection. The percentage of HepG2 and SMMC77221 cells in the $\mathrm{G}_{0} / \mathrm{G}_{1}$ phase $(63.9 \pm 2.6$ and $64.57 \pm 1.9$, respectively) of the lenti-siRNA/PHP14-transfected group was significantly 
A

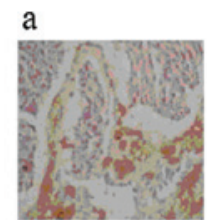

HCC tissues b

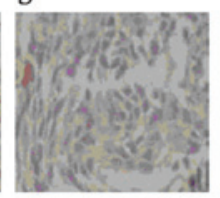

Non-cancerous region of $\mathrm{HCC}$

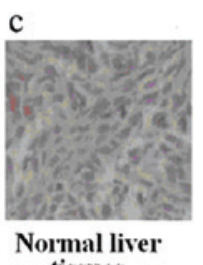

tissues
C

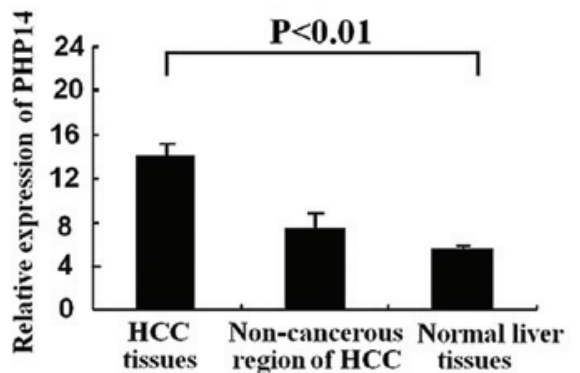

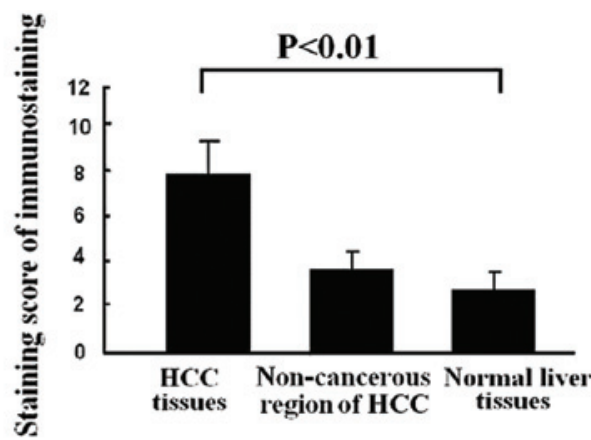

D

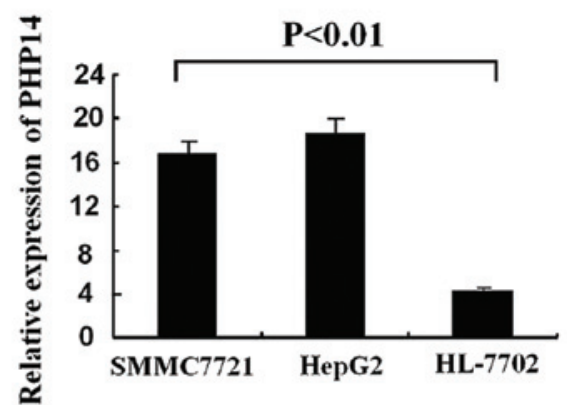

E

HepG2 SMMC7721 HL-7702

PHP14

$\beta$-actin

Figure 1. Immunohistochemistry, western blot analysis and RT-PCR analysis of PHP14 protein and mRNA expression in HCC tissues and cell lines. (A) Immunohistochemical staining of PHP14 protein in HCC tissues, adjacent non-cancerous tissues and normal liver tissues (magnification, x400). (B) Immunostaining scores in the HCC tissues were higher compared with those in the adjacent non-cancerous tissues and normal liver tissues. (C) Expression of PHP14 mRNA in HCC tissues was significantly higher compared with the adjacent non-cancerous tissues and normal liver tissues. (D) PHP14 protein expression was markedly increased in HCC cell lines, SMMC7721 and HepG2, compared with the human liver cell line, HL-7702. $\beta$-actin expression levels were used as an internal control. (E) Expression of PHP14 mRNA in HepG2 and SMMC7721 cells were significantly higher than those in HL-7702 cells $(\mathrm{P}<0.01)$. HCC, hepatocellular carcinoma; PHP14, 14-kDa phosphohistidine phosphatase. RT-PCR, reverse-transcription polymerase chain reaction.

higher compared with that in the src-siRNA-transfected group $(53.56 \pm 2.3$ and $54.26 \pm 2.5$, respectively; $\mathrm{P}<0.01)$. However, the percentage of HepG2 and SMMC7721 cells in the S phase $(13.74 \pm 2.5$ and $15.89 \pm 2.3$, respectively) of the lenti-siRNA/PHP14-transfected group was significantly lower compared with that in the src-siRNA-transfected group (19.22 \pm 1.6 and 19.52 \pm 1.4 , respectively) $(\mathrm{P}<0.01$; Fig. 3D).

Collectively these data indicate that lenti-siRNA/PHP14 exhibited a specific inhibitory effect on HepG2 and SMMC7721 cell growth. They also suggest that lenti-siRNA/PHP14 induces $G_{0} / G_{1}$ phase arrest and inhibits $S$ phase entry.

Lenti-siRNA/PHPI4 induces cell apoptosis in HCC cells. The effect of lenti-siRNA/PHP14 on the apoptosis of HCC cells was investigated using flow cytometry (Fig. 4A). It was identified that $8.91 \%$ of the HepG2 cells transfected with src-siRNA were apoptotic, while $17.82 \%$ of the HepG2 cells transfected with lenti-siRNA/PHP14 were apoptotic. We also identified that $8.23 \%$ of the SMMC7721 cells transfected with src-siRNA were apoptotic, while $15.98 \%$ of the SMMC7221 cells transfected with lenti-siRNA/PHP14 were apoptotic. These data suggest that knockdown of PHP14 by lenti-siRNA/PHP14 specifically induces apoptosis of the PHP14-overexpressing HCC cell lines, HepG2 and SMMC7721 (Fig. 4B).
A

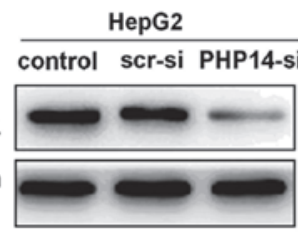

C

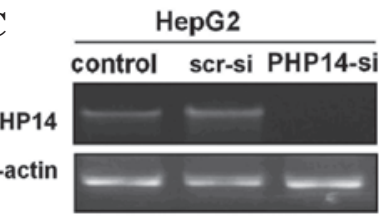

B

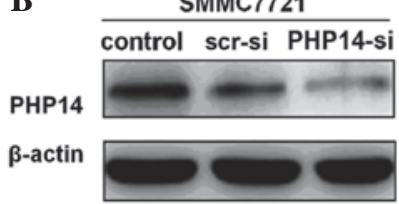

D

SMMC7721

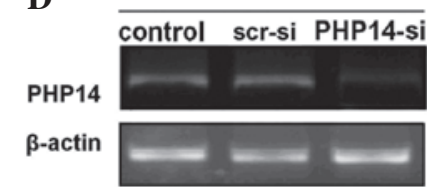

Figure 2. Effects of lentivirus-mediated knockdown of PHP14 expression in HepG2 and SMMC7721 cells. Lentivirus-delivered vectors for PHP14-siRNA (PHP14-si) were constructed and were demonstrated to be specific and potent in silencing PHP14 expression in the HCC cell lines, HepG2 and SMMC7721. Western blot analysis of PHP14 protein expression in (A) HepG2 cells and (B) SMMC7721 cells transfected with the lenti-siRNA/PHP14 vectors or the src-siRNA vector. RT-PCR analysis of PHP14 mRNA levels in (C) HepG2 cells and (D) SMMC7721 cells transfected with the lenti-siRNA/PHP14 vectors or the src-siRNA vector. scr-si (scr-siRNA), lentivirus-delivered scramble small interfering RNA vector; PHP14-si (PHP14-siRNA and lenti-siRNA/PHP14), lentivirus-delivered 14-kDa phosphohistidine phosphatase-specific small interfering RNA vector. HCC, hepatocellular carcinoma; RT-PCR, reverse-transcription polymerase chain reaction. 


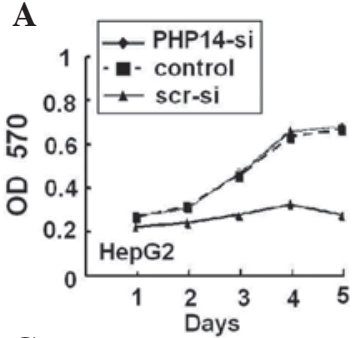

C

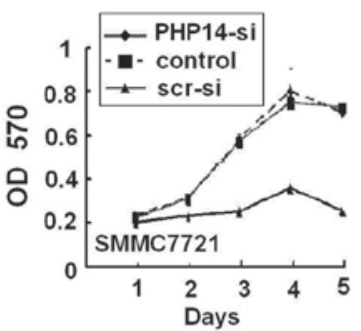

HepG2
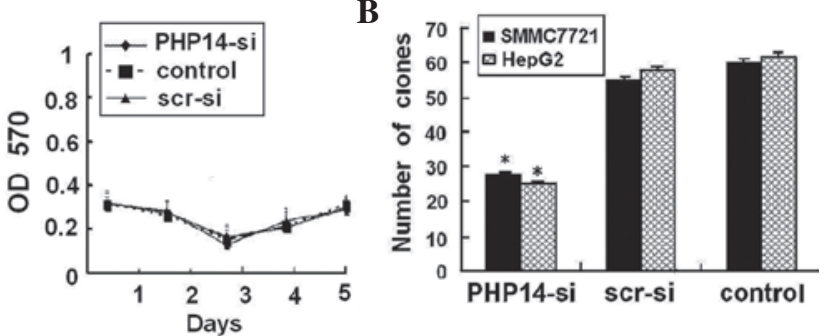

scr-si

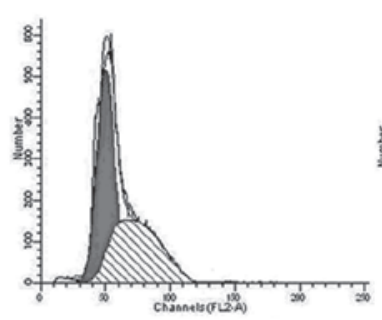

PHP14-si

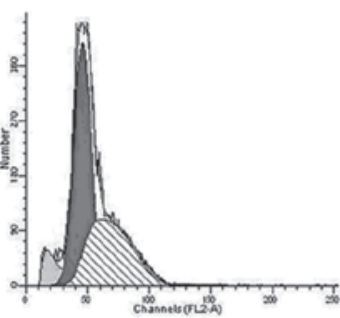

SMMC7721

scr-si

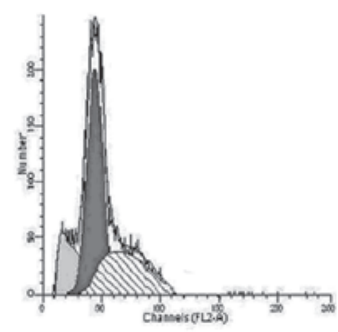

PHP14-si

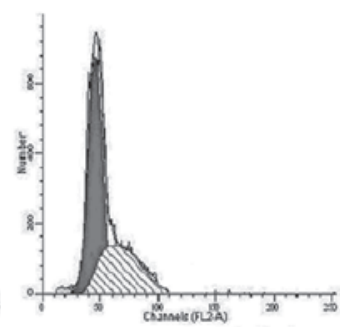

D

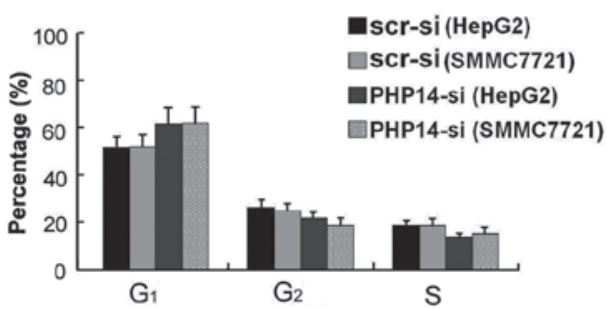

Figure 3. Lenti-siRNA/PHP14 vectors inhibited HCC cell growth and S phase entry. (A) The growth of HCC cells and normal liver cells transfected with the lenti-siRNA/PHP14 vectors was measured using an MTT assay. Values are presented at the indicated time points as the mean absorbance with a standard deviation of 3 wells. (B) A colony formation assay was conducted using HepG2 and SMMC-7721 cells infected with the virus for $96 \mathrm{~h}$. The cells without any infection were the control group. The data are averages from 3 independent triplicate experiments ( $<<0.01$ vs. control). (C) Lenti-siRNA/PHP14 decreased $\mathrm{S}$ phase population in HCC cells after infection for $96 \mathrm{~h}$. The $\mathrm{S}$ phase population in the indicated cells was determined using flow cytometry. (D) Flow cytometry demonstrated that knockdown of PHP14 expression via the lenti-siRNA/PHP14 induced $\mathrm{G}_{0} / \mathrm{G}_{1}$ phase arrest and decreased the S phase cell population, indicating a disruption in cell cycle progression. OD, optical density; PHP14-si (lenti-siRNA/PHP14), lentivirus-delivered 14-kDa phosphohistidine phosphatase-specific small interfering RNA vector; scr-si (scr-siRNA), lentivirus-delivered scramble small interfering RNA vector. HCC, hepatocellular carcinoma; MTT, 3-(4,5-dimethylthiazole-2-yl)-2,5-biphenyl tetrazolium bromide.

A

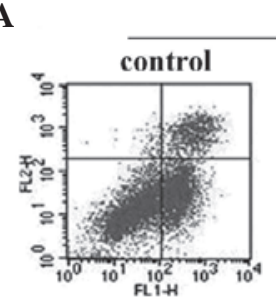

HepG2
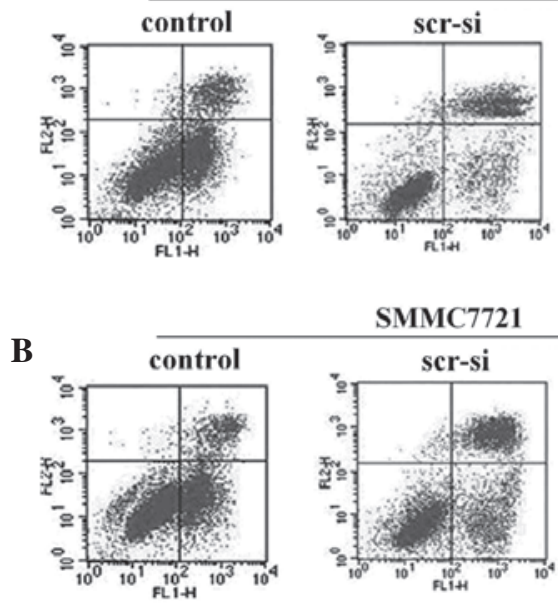

SMMC7721

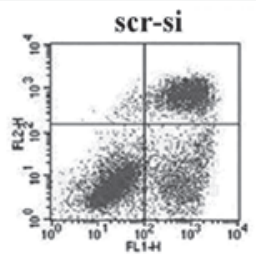

PHP14-si
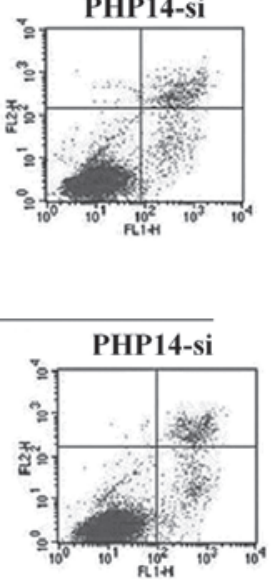
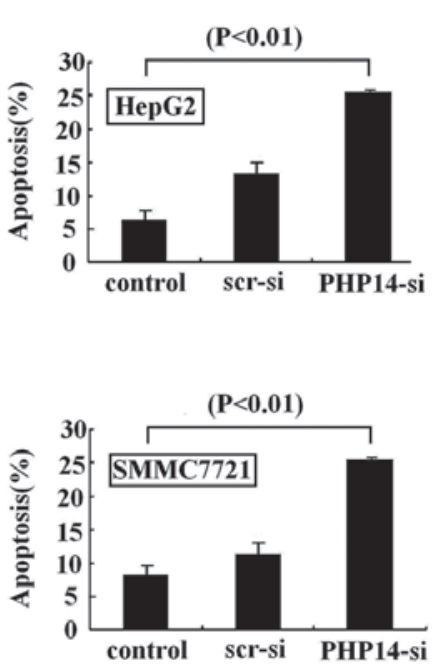

C
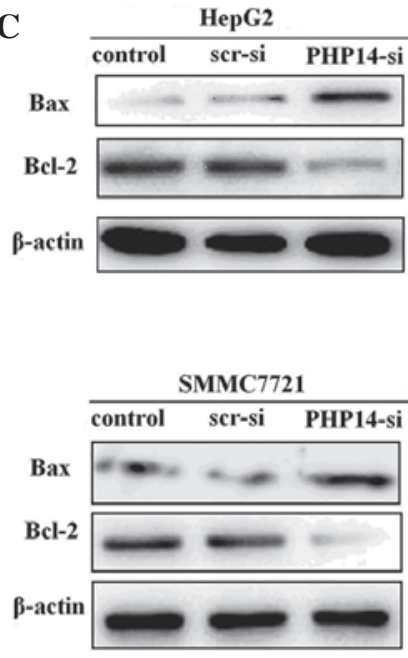

Figure 4.Lenti-siRNA/PHP14 vectors promote HCC cell apoptosis. Flow cytometry demonstrated that knockdown of PHP14 expression via lenti-siRNA/PHP14 increased (A) HepG2 cells apoptosis and (B) SMMC7721 cell apoptosis. (C) Western blot analysis of Bcl-2 family mediators of apoptosis revealed that knockdown of PHP14 resulted in downregulation of Bcl-2 and upregulation of Bax in lenti-siRNA/PHP14-transfected HepG2 and SMMC7721 cells, suggesting that the apoptotic effect of PHP14 may be partly mediated by Bcl-2 family member proteins. scr-si (scr-siRNA), lentivirus-delivered scramble small interfering RNA vector; PHP14-si (lenti-siRNA/PHP14), lentivirus-delivered 14-kDa phosphohistidine phosphatase-specific small interfering RNA vector. $\mathrm{HCC}$, hepatocellular carcinoma. 
In order to explore the underlying molecular mechanism of PHP14 and cell apoptosis, we analyzed the Bcl-2 family mediators of apoptosis, including Bcl-2 and Bax, using western blot analysis. The results indicated that knockdown of PHP14 downregulated Bcl-2 and upregulated Bax expression in lenti-siRNA/PHP14-transfected cells, suggesting that the apoptotic effect of PHP14 may be partly mediated by these Bcl-2 family proteins (Fig. 4C).

\section{Discussion}

$\mathrm{HCC}$ is an aggressive malignant tumor with high incidence and poor prognosis, particularly in Asia and Africa (3). Previous studies have demonstrated that the incidence of HCC in the USA and the UK has increased in past 2 decades $(22,23)$. Although varies therapies for HCC are available, the recovery rate remains unsatisfactory. Thus, an effective treatment method for $\mathrm{HCC}$ is urgently required.

PHP14 was the first histidine phosphatase protein identified in vertebrates, and is similar to the janus proteins of Drosophila $(12,13)$. PHP14 and its role in human lung cancer cell migration and invasion has been identified (19). PHP14 may play a role in neuronal signaling transduction; however, the role of PHP14 in other cancers remains unknown.

In this study, we demonstrated that PHP14 expression is elevated in the majority of HCC patient samples and HCC cell lines. Consequently, we hypothesized that the knockdown of PHP14 may inhibit tumorigenic growth of HCC in which PHP14 is overexpressed.

Using immunohistochemistry, we studied the PHP14 expression of $38 \mathrm{HCC}, 37$ adjacent non-cancerous and 31 normal hepatic tissues. Using western blot analysis and RT-PCR, we identified that PHP14 protein and mRNA are highly expressed in HCC tissues compared with adjacent non-cancerous tissue. The average staining score in HCC tissues was significantly higher compared with that in adjacent non-cancerous and normal hepatic tissue. This suggested that PHP14 plays a role in HCC carcinogenesis and may have a facilitative effect on the proliferation of HCC cells. Using western blot analysis, we demonstrated that PHP14 expression was upregulated in HCC cell lines compared with the normal liver cell line. Our study also confirmed that PHP14 was upregulated in HCC.

To determine whether the ectopic expression of PHP14 is able to modulate the proliferation of HCC cells, lentivirus vector siRNAs targeting PHP14 were transfected into HepG2 and SMMC7721 cells. Lentivirus, a type of reversal virus, is able to integrate into the host genome and demonstrate long-term expression of integrated genes (24). Lentivirus has a higher titer than a retroviral virus and does not only infect dividing phase cells, but also infects non-dividing cells. Using lentivirus vectors, one pair of siRNAs targeting PHP14 were transfected into HepG2 and SMMC7721 cells. As a result, we revealed that PHP14-si significantly inhibited HCC cell proliferation and growth in vitro. The stimulatory effects of PHP14 on HepG2 and SMMC7721 cells demonstrated that the PHP14 gene may be a growth promoter gene that acts directly or indirectly to control the proliferation of cells. The products of such genes regulate cell growth and differentiation in a positive way and thus promote neoplastic development, which further indicates that PHP14 may act as a tumor promoter in
HCC. These result are different from those of Xu et al who suggested that PHP14 expression had no effect on lung cancer cell growth and proliferation (19).

We also used flow cytometry to examine how PHP14-si affects the cell cycle. Once cells were treated with PHP14-si for $96 \mathrm{~h}$, we observed a significant increase of G1 phase population in all HCC cells. This result indicated that the knockdown of PHP14 expression inhibited HCC cell proliferation, which is necessary for entering into the $\mathrm{S}$ phase.

In this study, we examined the expression level of two Bcl-2 family members, Bcl-2 and Bax, in this PHP14 depletion-triggered apoptosis in $\mathrm{HCC}$ cells. We identified that the knockdown of PHP14 downregulated Bcl-2 and upregulated Bax in PHP14-siRNA-transduced tumor cells, suggesting that Bcl-2 family members may partially contribute to the apoptosis of HCC cells with PHP14 depletion.

In conclusion, PHP14 may be an effective gene target for HCC treatment. The results of this study suggested that the significant downregulation of PHP14 expression by lenti-siRNA/PHP14 in HCC cells results in the inhibition of $S$ phase entry, inhibition of cell proliferation and increased cell apoptosis. Therefore, knockdown of PHP14 using lentivirus-delivered siRNA may be a valuable approach for the treatment of HCC. The correlation between PHP14 and HCC cell invasion and metastasis requires further study.

\section{References}

1. Jemal A, Siegel R, Ward E, Hao Y, Xu J, Murray T and Thun MJ: Cancer statistics, 2008. CA Cancer J Clin 58: 71-96, 2008.

2. El-Serag HB, Davila JA, Petersen NJ and McGlynn KA: The continuing increase in the incidence of hepatocellular carcinoma in the United States: an update. Ann Intern Med 139: 817-823, 2003.

3. Parkin DM, Bray F, Ferlay J and Pisani P: Global cancer statistics, 2002. CA Cancer J Clin 55: 74-108, 2005.

4. Jemal A, Thomas A, Murray T and Thun M: Cancer statistics, 2002. CA Cancer J Clin 52: 23-47, 2002.

5. Llovet JM, Burroughs A and Bruix J: Hepatocellular carcinoma. Lancet 362: 1907-1917, 2003.

6. Colombo M: Hepatocellular carcinoma. J Hepatol 15: 225-236, 1992.

7. Lai EC, Fan ST, Lo CM, Chu KM, Liu CL and Wong J: Hepatic resection for hepatocellular carcinoma. An audit of 343 patients. Ann Surg 221: 291-298, 1995.

8. Takenaka K, Kawahara N, Yamamoto K, et al: Results of 280 liver resections for hepatocellular carcinoma. Arch Surg 131: 71-76, 1996.

9. Bruix J and Llovet JM: Prognostic prediction and treatment strategy in hepatocellular carcinoma. Hepatology 35: 519-524, 2002.

10. Qian C, Drozdzik M, Caselmann WH and Prieto J: The potential of gene therapy in the treatment of hepatocellular carcinoma. J Hepatol 32: 344-351, 2000.

11. Ruiz J, Qian C, Drozdzik M and Prieto J: Gene therapy of viral hepatitis and hepatocellular carcinoma. J Viral Hepat 6: 17-34, 1999.

12. Ek P, Pettersson G, Ek B, Gong F, Li JP and Zetterqvist O: Identification and characterization of a mammalian 14-kDa phosphohistidine phosphatase. Eur J Biochem 269: 5016-5023, 2002.

13. Klumpp S, Hermesmeier J, Selke D, Baumeister R, Kellner R and Krieglstein J: Protein histidine phosphatase: a novel enzyme with potency for neuronal signaling. J Cereb Blood Flow Metab 22: $1420-1424,2002$

14. Klumpp S and Krieglstein J: Reversible phosphorylation of histidine residues in vertebrate proteins. Biochim Biophys Acta 1754: 291-295, 2005.

15. Klumpp S, Bechmann G, Maurer A, Selke D and Krieglstein J: ATP-citrate lyase as a substrate of protein histidine phosphatase in vertebrates. Biochem Biophys Res Commun 306: 110-115, 2003. 
16. Maurer A, Wieland T, Meissl F, et al: The beta-subunit of G proteins is a substrate of protein histidine phosphatase. Biochem Biophys Res Commun 334: 1115-1120, 2005.

17. Klumpp S and Krieglstein J: Phosphorylation and dephosphorylation of histidine residues in proteins. Eur J Biochem 269: 1067-1071, 2002.

18. Steeg PS, Palmieri D, Ouatas T and Salerno M: Histidine kinases and histidine phosphorylated proteins in mammalian cell biology, signal transduction and cancer. Cancer Lett 190: 1-12, 2003.

19. Xu A, Hao J, Zhang Z, et al: 14-kDa phosphohistidine phosphatase and its role in human lung cancer cell migration and invasion. Lung Cancer 67: 48-56, 2010.

20. Shen C, Buck AK, Liu X, Winkler M and Reske SN: Gene silencing by adenovirus-delivered siRNA. FEBS Lett 539: 111-114, 2003.
21. Maaser K, Daubler P, Barthel B, et al: Oesophageal squamous cell neoplasia in head and neck cancer patients: upregulation of COX-2 during carcinogenesis. Br J Cancer 88: 1217-1222, 2003.

22. El-Serag HB and Mason AC: Rising incidence of hepatocellular carcinoma in the United States. N Engl J Med 340: 745-750, 1999.

23. Taylor-Robinson SD, Foster GR, Arora S, Hargreaves S and Thomas HC: Increase in primary liver cancer in the UK, 1979-94. Lancet 350: 1142-1143, 1997.

24. Pfeifer A, Lim T and Zimmermann K: Lentivirus transgensis. Methods Enzymol 477: 3-15, 2010. 